\title{
Health care policy and regulatory implications on medical device innovations: a cardiac rhythm medical device industry perspective
}

\author{
Arjun Sharma - Anthony Blank • Parashar Patel • \\ Kenneth Stein
}

Received: 2 July 2012 / Accepted: 13 January 2013 /Published online: 10 March 2013

(C) The Author(s) 2013. This article is published with open access at Springerlink.com

Keywords Medical devices · Regulatory barriers · Regulatory costs · Comparative effectiveness

\section{Introduction}

"How wonderful that we have met with a paradox. Now we have some hope of making progress." - Niels Bohr [1]

The medical technology industry in the early twenty-first century is marked by a puzzling paradox. The industry has contributed to decades of life-saving innovations: the introduction of technologies like the implantable cardioverter defibrillator, radiofrequency catheter ablation for cardiac arrhythmias, and cardiac resynchronization therapy, and we have witnessed remarkable reductions in mortality and morbidity due to cardiovascular disease [2, 3]. Nevertheless, we are simultaneously witnessing escalating concerns from politicians, the plaintiff's bar, the general public, physicians, researchers, and patients over whether our technologies truly are safe and effective [4-6]. Behind this paradox lies another, more subtle paradox: a vigorously innovative medical technology industry requires strong and complex underpinnings, but there is a growing sense that overly burdensome and complex regulations threaten to choke off continued innovation. Our intent is to explore this paradox, by expressing the authors opinions based on their medical

A. Sharma $\cdot$ A. Blank $\cdot$ P. Patel $\cdot$ K. Stein $(\triangle)$

Boston Scientific Corporation, 4100 Hamline

Ave North, MS 3-200,

St. Paul, MN 55102, USA

e-mail: kenneth.stein@bsci.com device industry viewpoint, focusing on three areas of concern: the threat posed by a globally discordant patchwork of different regulatory approaches and standards, the threat posed by a particularly burdensome regulatory process within the USA, and the implications of inappropriate use of comparative effectiveness with respect to medical devices.

\section{The global environment for innovation: regulatory discord or harmony}

The requirements to introduce new medical devices can vary dramatically around the world. For example - consider the differences between the USA and the European Union. In the USA, the foundations of the regulatory requirements for medical products are defined statutorily in the Federal Food, Drug and Cosmetic Act and implemented in the Code of Federal Regulations [7]. Per this construct, higher risk devices (e.g., U.S. Class II and Class III) may only enter the US market after they have been demonstrated to provide a reasonable assurance of safety and effectiveness for the intended use in the labeled intended patient population. In contrast, under the European regulatory framework, manufacturers must establish the safety and performance of the proposed device - a subtly different standard (Council Directive 93/42/EEC) [8]. Furthermore, despite the similarity in the nomenclatures used for classification in the USA and the EU, the criteria used to classify, and thus identify those devices which must meet higher premarket thresholds, are quite different in these two regions. The net result of both these factors can be dramatically different requirements to place new medical technologies into the market for use with patients. There are two very different (and valid) perspectives 
on this consequence- - either that the patients in the region for which the regulatory requirements were lower (typically the EU) are benefitting from earlier access to new lifesaving technologies or that the patients in the region for which the requirements were higher are benefitting from access to technologies that have been more thoroughly vetted and-by extension - are either safer or more effective [9]. There are oft-cited examples to support either perspective - the early experiences with first-generation abdominal aortic aneurysm stent grafts being an example of the latter and the recent experiences with transcatheter aortic valve replacement (TAVR) devices appear to be emerging examples of the former. Regardless of the perspective, one fact remains indisputable: the discordant regulatory thresholds for medical devices to enter the market in countries around the world continue to provide financial and logistical challenges to the organizations trying to develop and make available new lifesaving and lifealtering technologies to physicians and patients. It also potentially creates challenges for patients moving from one regulatory region to another.

To further complicate the tapestry of regulatory requirements around the world, the impact of individual country regulatory requirements can have dramatic implications to the availability of new devices. In the medical devices space, this is manifested in the requirement that devices be subjected to localized versions of international performance standards - essentially requiring that the same type of testing be repeated to support local approval of the technology. Of greater impact is the growing insistence by regulators that clinical trials (premarket and/or post-market) be conducted either in whole or in part within a particular country. When one considers the growing number of regions (Brazil, Japan, India, and China) in which local requirements are emerging, the cost implications to manufacturers (and thus their ability to market their devices globally) are staggering. While there may be a rationale for testing pharmaceuticals in genetically diverse populations, clinical trials with cardiac rhythm management (CRM) devices in multiple countries has less merit, and rarely can these trials be statistically powered to truly answer implicit concern of geographically diverse response to therapy as the number of devices implanted in many of these countries, even post market, is often too low.

Industry representatives and regulators from countries around the world have met through the Global Harmonization Task Force (GHTF) in an effort to bring some modicum of harmony to the standards and requirements across the globe [10]. Despite tremendous effort and engagement from multiple stakeholders from around the world, there remains much to do if we are to inject a level of consistency, which materially impacts the consistency and global availability of impactful new medical technologies. The impacts of recent changes made to the organization of GHTF (renamed to IMDRF-
International Medical Device Regulators' Forum) remain to be seen.

\section{The US regulatory framework and innovation}

It would be impossible to proceed without addressing the growing perception by many key stakeholders (including industry and physicians) that FDA has become a barrier to medical device innovation and - either by action or inaction - is driving innovation outside of the USA. To explore this thesis, it is important to consider the foundations of the current regulatory process in the US. Congress writes acts, which the President signs into law, and which federal agencies, such as the FDA, interpret and write regulations, and which are available for a comment period prior to implementation. FDA regulations are contained in Title 21 of the Code of Federal Regulations [11, 12]. Specifically, the regulations represent FDA's interpretation of what the law (Federal Food, Drug and Cosmetic Act) tells them to do, and guidance documents may be written by the FDA to aid the public to comply with the intent of the regulations. It is premature for the authors to comment at this time on the practical impact of the recent Food and Drug Administration Safety and Innovation Act of July 2012, which includes the Medical Device Fees Act (MDUFA III) for the next 5 years [13]. Although there have been few changes in either the law or regulations which define the manner in which medical devices are to be regulated in the USA, there is the persistent belief the requirements have significantly changed.

To appreciate how this might be so, one can look at the regulatory standard for market entry - "reasonable assurance of safety and effectiveness." While simple in concept, the vagueness of this standard is embodied in that which constitutes "reasonable assurance" as defined by the expectations of the American public and as reflected by the actions of their representatives in government. Although the medical devices developed in this country have grown ever more complex to treat an ever increasingly complex set of patient conditions, there has been a seemingly simultaneous increase of expectations from the American public (and their representatives) of the reliability and safety of these same devices. In so doing, we have in essence shifted the definition of "reasonable assurance" in a direction which demands that the level of assurance that is "reasonable" is higher today than it was several years ago. Said differently, the American public has expressed a lowered threshold of risk they are willing to accept for a particular patient benefit in even the newest of technologies which treats the sickest of patients (one need only review the record of Congressional hearings during the past 3 years for evidence of this shift) [4]. In response to this, regulators have in essence "raised the bar" on the level of pre-market data (bench top, animal, and clinical) and post-market studies required for the approval of medical devices in the USA. 
Approval in the European Union is based primarily on thorough bench testing, engineering analysis, and post-market clinical data. However, while this used to be similar in the USA, the pre-market and post-market clinical requirements have increased in the last 10 years in the USA. For example, a US implantable cardioverter defibrillator (ICD) lead approval pre-market patient study has gone from 24 patient-years in 1998 to about 400 patient-years in 2008, and currently in 2012 to 1,000 patient-years, a 40 -fold increase (Fig. 1). In addition, in 2008, a post-approval study was added for about 1,800 patients enrolled with 5-year follow-up on 1,000. With a device sales life of 3-5 years, this represents a significant enough increase on the cost to obtain US market approval compared to European approval, to make commercialization of some devices unattractive in the USA (manufacturer data).

One may question whether CRM device reliability is worsening and necessitates this increased scrutiny. Defining device quality and reliability may be challenging as there are many potential measures, with each measure subject to variation unrelated to device. Maisel and colleagues [14] suggested that while pacemaker malfunctions had declined during the period of 1990-2001, ICD malfunctions had not declined. While those data were applicable to that time period, it should be considered that ICD therapy was in its infancy (FDA approval 5 years earlier 1985), and that time period saw the evolution of the ICD from a shock device to one with dual-chamber pacing and anti-tachycardia pacing. In contrast, pacemakers were at that time already a relatively mature technology. Maisel et al. [14] observed the malfunction rate for ICDs to be in the range of $1-4 \%$. Current ICDs appear to have fewer malfunctions, as reported by manufacturers' product performance reports, in the range of $1 \%$ at 5 years, despite improved device internal diagnostics to identify malfunction. A recent FDA study from 2003 to 2007 used annualized ICD and CRT-D explantation rates (AER) as an estimate of device reliability and malfunction. They concluded that in this more recent time period, there had been a favorable decline in the AER $(p<0.001)$,

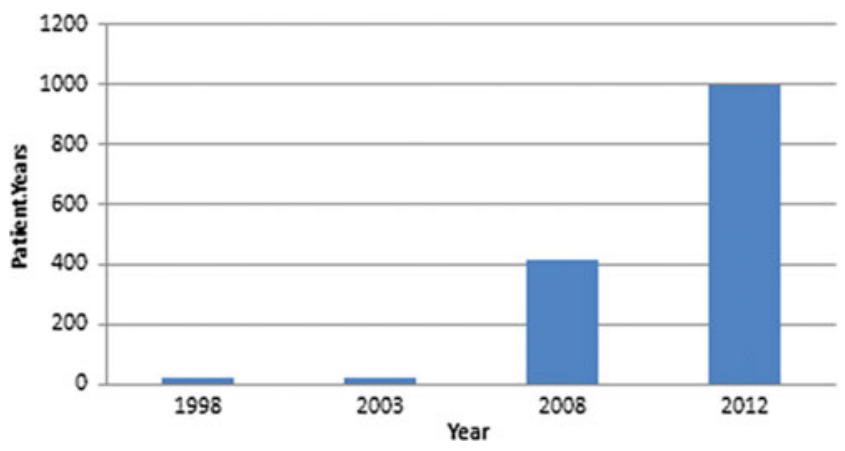

Fig. 1 Pre-market clinical testing required for FDA approval of ICD leads for one manufacturer, expressed in patient-years. This demonstrates an increase in the time and number of patients needed for study after 2007 (internal data, but available at the FDA) which might suggest that there is less device malfunction [15]. This decline is all the more significant when one considers the improved diagnostic capabilities of today's ICDs which allow the differentiation, for example, of normal battery depletion from component malfunction.

While this shift in standard may ultimately increase the safety and reliability of first-generation devices introduced in the USA, we are currently witnessing a decided shift of medical device manufacturers to focus their development and approval efforts either initially or exclusively to regions outside the USA [9]. While US approval often represents the de facto "gold standard" and provides great assurance to many, the cost to reach this objective has become increasingly prohibitive for many innovators. Thus, it is not unreasonable to project that access to new technologies in the USA will increasingly lag (perhaps by many years) the availability of these technologies outside the USA. While tempting to demonize the FDA as the cause of this shift, it is more likely that the agency and its leadership are not the cause of this, but are in many ways reacting to what the American public (and Government) is asking of them. In fact, the authors have found the FDA to be very helpful in working within this restrictive government policy framework, to collaboratively arrive at a trial design expected to meet the regulatory requirements and best serve patients.

Although challenging, there are signs that all is not lost. There seems now to be a growing recognition by government officials that the shifts in expectations are driving innovative medical technology (and the people that develop them) outside of the USA. The FDA has recently published new and revised Guidance documents which speak of their expectations for conducting feasibility clinical studies in the USA and the expectations and methodologies related to the assessment of risks and benefits [16].

It is this latter topic (risk and benefits) which will ultimately define the intersection of medical device regulation and innovation in this country. For one of the foundations of medical product law is the premise that no medical product - either drug or device - is without risk. The degree of risk we are willing to accept for a given benefit will ultimately define the breadth and type of pre-market evaluation (and the results) that must be completed before a product can enter the market.

Finally, incorporated into this analysis must be an honest discourse about the adoption of new medical technologies by the physician community. With each new innovative medical technology, there seems to be a predictable cycle of cautious adoption within the approved patient indications until comfortable with the technology. Once comfort has been attained, this is often followed by over-adoption, sometimes outside the approved (and studied) patient populations. This inevitable unbridled optimism driven by confidence in the technology (rather than assurance in the data) certainly feeds into the argument that more pre-market data 
collection (not less) is necessary to ensure patient safety. However, ultimately, physician bodies will need to determine appropriate device indications based on the greater experience post-market approval, potentially through guidelines. It should also be considered that off-label pharmaceutical use has led to some important discoveries such as beta blockers for heart failure, or ASA for stroke prevention. It is certainly possible that similar discoveries might occur later in the device area also.

Another component of regulatory oversight involves post-market surveillance and actions taken by manufacturers with fielded products. In the past decade, there have been many product field actions in the CRM area despite the largely unrecognized fact that performance of these devices (in terms of reliability and safety) has actually improved. Manufacturers typically execute these field actions voluntarily to reduce the risk of patient harm when either (1) a new and previously unrecognized hazard associated with the device or its use has emerged or (2) a hazard has emerged with a greater frequency or harm than previously recognized or expected. Manufacturers typically become aware of these malfunction events through physician and patient complaints captured and analyzed in their post-market surveillance systems. The apparent paradox of more advisories despite improving overall performance is consistent with a lower tolerance of malfunctions at multiple levels. Thus, a recent study of 1,644 consecutive ICD implants between 1996 and 2004 revealed that 704 patients(43\%) were part of an advisory, but only 28 of 1,644 patients (1.7\%) actually had an advisory-related device malfunction, and Class I recalls were not associated with increased mortality [17]. Some patients did undergo device replacement $(42 / 1,644$, $2.5 \%$ ) and hence were harmed, but it should also be considered that ultimately, if the patient does not succumb to comorbidities, that most ICDs need to be replaced and that this is a fractional harm depending on the device age when replaced.

Field actions taken by a manufacturer are often very expensive and come with an attendant amount of attention, publicity, and legal action. While this attention provides significant opportunities to inform physicians and patients, it often leads to fear and-sometimes-inappropriate actions. Significantly, some field actions have been for malfunctions that are uncommon and occur in products with an overall performance that is considered acceptable [18]. In 2004, Maisel [19] found that many physicians would explant a pacemaker if it had a malfunction rate of $1 / 10,000$, which is notably a better overall performance than any known pacemaker has ever demonstrated. There may now be a better physician understanding of the risks of device explantation. However, recently, when physicians have been faced with a situation where one of their patients has experienced a $1 / 10,000$ sporadic component malfunction, we have observed that sometimes there is still an initial physician response to want to explant all similar devices from their patients or to take special unnecessary precautions. In short, it appears that patient and physician expectations for the performance of CRM devices may have outstripped engineering capability, and this potentially leads to both patients and physicians failing to utilize beneficial device therapy or creating, secondarily through physician actions, more patient harm than the original device hazard [20]. While it is clear that the onus is on the industry to meet today's standards and expectations, it is also clear that those standards and expectations are becoming more stringent. A high profile example of this is an ICD lead recall from 2007 (6-year lead survival, $84.8 \%$ ). While that same manufacturer had a better immediately precedent product, the ICD lead made by the same manufacturer in the late 1990s had a similar malfunction rate (6-year lead survival, $86.8 \%$ ), and the product from the 1990s was not subject to any regulatory action because it met the performance expectations of a decade earlier. It must also be recognized that the lead recalled in 2007 was likely designed in around 2000, when the performance expectations were different [21].

An appropriately designed post-market surveillance program by medical device manufacturers, which captures unexpected hazards as well as appropriate device performance, is necessary to ensure the continued availability of medical devices in the USA. When performance does not meet regulatory expectations, executing a worldwide field action may cost a manufacturer over 50 million dollars, excluding the secondary loss of future business, and/or legal costs which are often far more. This fact has a potential significant effect on innovative technology. Manufacturers now must consider not only approval of a device but also the potential postmarket quality/regulatory risks any new innovative device may pose over time. Thus, some manufacturers chose to deliberately avoid the development of products with the greatest regulatory or liability risks. Although the US Supreme Court ruled that in Riegel v. Medtronic a medical device manufacturer cannot be sued under state law by patients alleging harm from a device that received marketing approval from the FDA, this does not end all legal actions, which also continue to be a significant cost to manufacturers, particularly in the USA. Thus, the post-market regulatory environment in the USA provides several significant disincentives to innovative technology. Consistent with this, there has been a steady decline in both $510(\mathrm{k})$ and PMA submissions to the FDA (Fig. 2) [22, 23]. This suggests that the current environment has already potentially significantly negatively impacted medical innovation despite the advances in genomics, nanotechnology, and computing power. However, there are also other significant non-regulatory disincentives currently to medical device innovation, particularly in the USA. These include the economic situation which has reduced available venture capital, concerns about the medical device excise tax, the projected reductions in health care spending as a disincentive as it 
Fig. 2 Progressive declines in 510(k) and PMA submissions to the FDA between 1999 and 2009. Reproduced from [22]

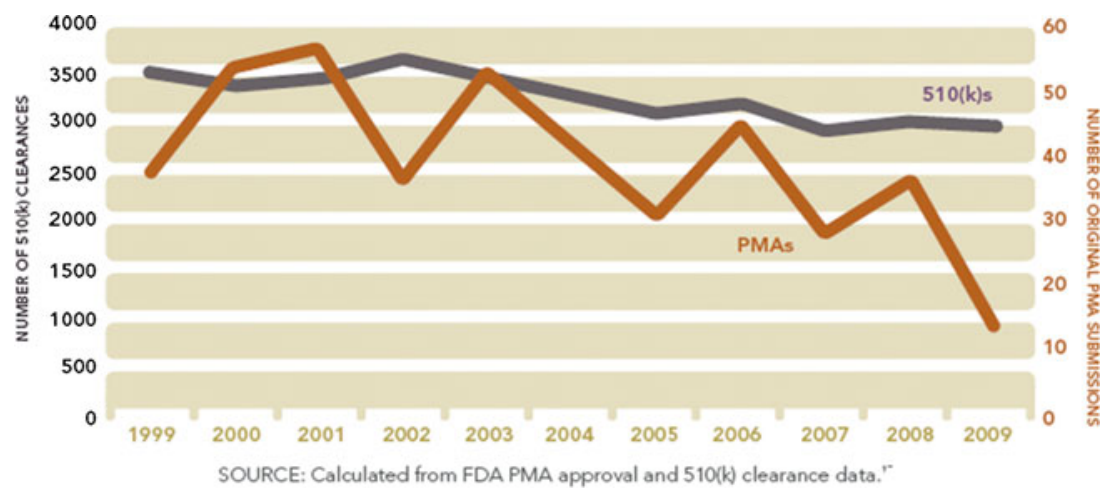

cancer screening in elderly men and the effectiveness and costs of osteoporosis screening and hormone replacement therapy [24].

In 2010, with the enactment of the Accountable Care Act, the federal government created the Patient-Centered Outcomes Research Institute (PCORI) whose mission is to help "people make informed health care decisions, and improve[s] health care delivery and outcomes, by producing and promoting high integrity, evidence-based information that comes from research guided by patients, caregivers and the broader health care community." PCORI funding was US $\$ 150$ million for fiscal year 2012 [25]. Needless to say, this represents a small fraction of government expenditures on health care research (as the NIH invests US $\$ 30.9$ billion annually in medical research) [26]. PCORI is not the only federal entity active in CER. The Agency for Health Care Quality and Research undertakes CER-like activities (e.g., technology assessments), frequently at the request of the Centers for Medicare and Medicaid Services (CMS). States are also actively considering or conducting CER, with the State of Washington's Health Technology Assessment program being the most visible and notable example. Perhaps the best-known agency is the United Kingdom National Institute for Clinical and Health Excellence (NICE), which conducts CER and disseminates its findings to patient, clinicians, purchasers of care, and others to inform decision making. Other organizations, such as the Blue Cross and Blue Shield Technology Evaluation Center, and private payers also engage in technology assessment for use in making coverage decisions. Health technology assessment is actively performed in France, Germany, Belgium, Italy, and Australia, with other countries actively considering establishing similar programs (South Korea and Japan, for example).

Industry supports CER, provided the focus is on the clinical appropriateness of alternative treatments, and the research agenda and related questions are shaped with input from multiple stakeholders, including physician specialty societies and medical device companies. To be most effective, conducting CER and interpreting results in the context of medical devices, policy makers, physicians, and issued CER reports on the cost effectiveness of prostate 
Table 1 Individual trial data demonstrating the impact of follow-up time on observed benefit (life-years gained per device implanted) and on observed number needed to treat to gain 1 life-year

Reproduced from [15]

\begin{tabular}{|c|c|c|c|c|c|c|}
\hline \multirow[t]{2}{*}{ Trial } & \multicolumn{3}{|c|}{$\begin{array}{l}\text { Life-years gained per } \\
\text { device implanted }\end{array}$} & \multicolumn{3}{|c|}{$\begin{array}{l}\text { Size of number needed to } \\
\text { treat to gain } 1 \text { life-year }\end{array}$} \\
\hline & 1 year & 2 years & 3 years & 1 year & 2 years & 3 years \\
\hline Wever et al. [35] & 0.10 & 0.30 & 0.58 & 10 & 3.3 & 1.7 \\
\hline MADIT [36] & 0.11 & 0.30 & 0.50 & 9 & 3.3 & 2.0 \\
\hline AVID [37] & 0.05 & 0.12 & 0.22 & 21 & 8 & 4.6 \\
\hline CIDS [38] & 0.01 & 0.04 & 0.09 & 123 & 24 & 11 \\
\hline MADIT II [39] & 0.01 & 0.06 & 0.13 & 133 & 17 & 8 \\
\hline CASH [40] & 0.01 & 0.09 & 0.18 & 133 & 11 & 5.6 \\
\hline Schläpfer et al. [41] & 0.07 & 0.23 & 0.41 & 15 & 4.4 & 2.5 \\
\hline MUSTT [42] & 0.07 & 0.21 & 0.40 & 15 & 4.7 & 2.5 \\
\hline
\end{tabular}

purchasers should be cognizant of several factors that can influence CER findings.

Perhaps the most important factor is the time frame considered when studying implantable medical device interventions. For interventions that act continuously over time, as is the case for many implantable devices, the magnitude of clinical benefit increases with time. Thus, the benefit from the device increases with time and the number of patients needed to treat to save a life, for example, diminishes with longer follow-up. Table 1 shows the effect of varying follow-up duration on the number needed to treat to save a life with ICDs [27], and this is graphed in Fig. 3. Thus, a key question for those designing clinical studies is the length of follow-up. If it is too short, a CER review could result in an unfavorable conclusion. Lengthening the follow-up time, however, has consequences. The sponsor, frequently medical device companies, will incur higher costs. And importantly for patients, physicians, and company sponsors, depending on the study design, a longer follow-up could increase the time to market and prevent the technology from being available to patients quickly. In the past, CRM device

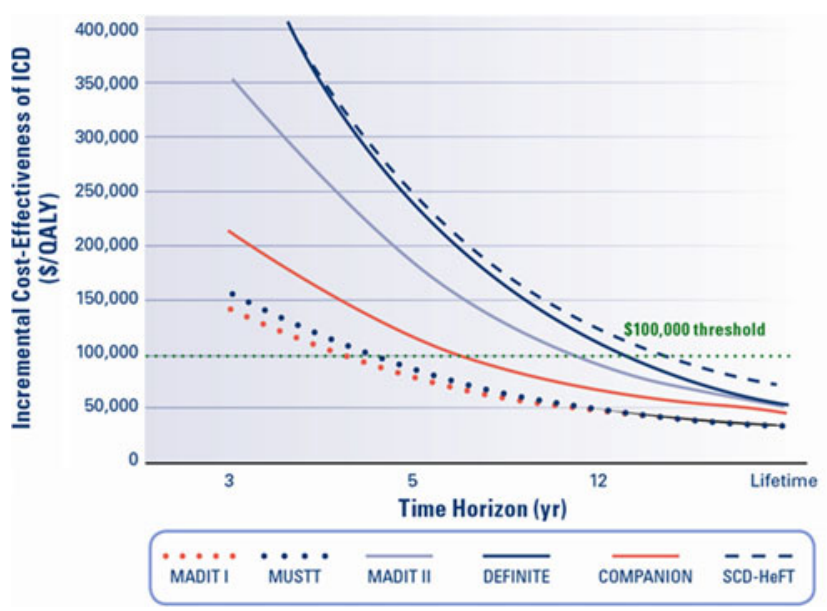

Fig. 3 Cost effectiveness ICD versus control over lifetime in 2005 US dollars manufacturers have tended to focus on the comparison of device versus optimal pharmaceutical therapy for the prevention of sudden death or treatment of heart failure, with the measured outcomes focused on mortality, heart failure hospitalization, and, more recently, on economic endpoints. In the future, it may be necessary to focus on other outcomes and other comparative treatment strategies.

Time also plays an important role with respect to cost effectiveness reviews. Using the example in the table, making conclusions about the relative benefit of an ICD implant at 1 year will yield different conclusions than when making conclusion 3 years after implant because of the up-front costs of the device and surgical implantation. The same issue must be considered when assessing the cost effectiveness of therapies. For example, the cost effectiveness of ICD therapies can range from about US $\$ 150,000$ to US $\$ 300,000$ at 3 years, but drop substantially to about US $\$ 50,000-100,000$ over a lifetime. Using a lifetime frame as the basis of evaluation yields a more appropriate understanding of the value of ICD therapies as, in general, ICD implantation is a lifelong treatment strategy, and MADIT II 8-year follow-up, for example, shows continuing benefit to the therapy over that extended time period [28]. However, over increasing time, there may emerge new comorbidities which can also affect outcomes not influenced by an ICD. Thus, consideration needs to be given to potential changing effectiveness of the device over time. An examination of the potential impact of comorbidities on the mortality of an initial implant ICD population was recently published [29], and this type of analysis may need to be considered over time, for example, with each subsequent device replacement.

A second important factor to consider is the evolutionary nature of medical device innovation. Medical device interventions frequently require time to evolve. Continuous evolution and improvement in the technology, procedure techniques, and physician skill will influence clinical outcomes and thus, in many cases, affect patient outcomes. As with the follow-up time, the evolutionary nature of medical device innovation 
must be considered both by trial sponsors as well as in the interpretation of results under CER. For example, the safety and efficacy endpoints for a particular left atrial occlusion device improved significantly over the course of just a few years with physician experience [30]. Furthermore, during the time course of a long-term CER, the medical device being assessed has often undergone production of new generations of products associated with improved outcomes. The challenge for policy makers is to balance between "too early to be evaluated appropriately" and such widespread dissemination that changing clinical practice is challenging even with an unfavorable finding of effectiveness. For example, the United States Preventive Services Task Force generated substantial controversy in October 2011 with its draft recommendation against PSA-based screening for prostate cancer. The recommendation was based on an update to a 2008 evidence synthesis which concluded that the evidence was insufficient to assess the balance of benefits and harms in men $<75$ years of age. The challenge for physicians, payers, and patients is that while the evidence was being generated, the use of PST-based screening became widespread, with over half of American men over 50 years of age receiving the test. A favorable recommendation is not a guarantee that clinical practice will change either. For example, despite the depth and quality of clinical data and a relatively favorable NICE guidance, ICD therapy use remains underutilized in England and Wales [31, 32].

A related challenge for CER will be to keep evidence reviews and technology assessments updated as technology evolves and clinical guidelines change. Policy makers and those conducing CER must balance resources between the need for evidence in virtually all clinical, therapeutic, and health care delivery spaces with the need to update existing research to keep pace with innovation. For example, while technology and clinical guidelines have changed with respect to cardiac resynchronization therapy, many coverage policies in the USA and NICE guidance remain unchanged (NICE is in the process of reviewing its guidance on ICD and CRT-Ds and expects to issue revised guidance in late 2013). Physicians and purchasers will have to consider the age of CER assessments with more current clinical data and clinical guidelines when making clinical choices and coverage decisions. Physicians will also have to consider that the rapid evolution in devices will mean that CER (and sometimes clinical guidelines) is based on technology that is several generations older than currently available.

The cycle of innovation and interaction with CER and guidelines may also require device companies to develop a range of post-market research strategies to ensure that the available data are consistent with later generations of technology. Payers will also undoubtedly use CER to inform coverage requirements. For example, CMS's recent decision to cover TAVR for Medicare patients will require hospitals and physicians to participate in a national registry that will follow patients for at least 1 year and answer specific questions on patient outcomes (e.g., stroke, mortality, etc.) and quality of life. Collecting and disseminating such data will increase costs for physicians, hospitals, and medical device companies. However, the rationale for such registries is in part based on the need to monitor the performance of complex devices in non-investigative centers [33].

Other factors to consider when designing comparative clinical studies and interpreting CER reviews are the patient population studied and the number of patients being studied. Clinical trials, by their nature, study average effects in large populations. Yet, when it comes to our own health, none of us are interested in what works best for the average patient-we all want to know what works best for the individual patient we are caring for at a given point in time. Making sure that comparative clinical studies are large enough to conduct meaningful subgroup analyses is therefore important. A good example comes from the MADIT-CRT Study. This trial-looking at the effect of cardiac resynchronization therapy in patients with minimally symptomatic congestive heart failure - met its primary endpoint for the entire population studied. However, subgroup analysis revealed a marked difference in outcome according to the presence or absence of left bundle branch block (Fig. 4). A CER review using the group average would understate the effect in the patients with left bundle branch block while overstating the effect in the remainder. Subgroup analysis may become particularly important in view of the

\section{Left Bundle Branch Block $(n=1281)$}

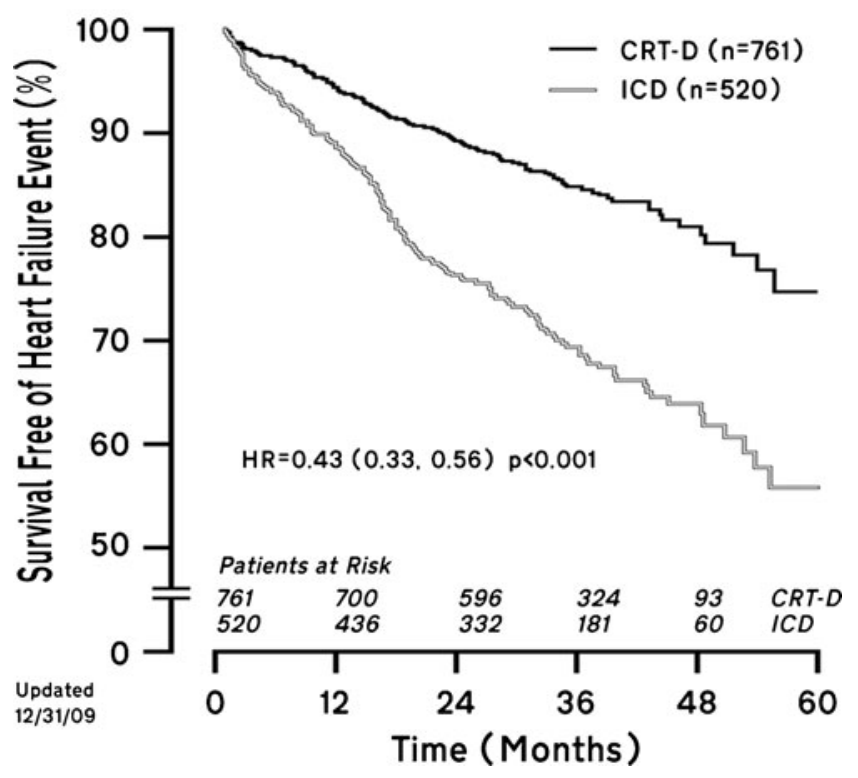

Fig. 4 MADIT-CRT post hoc analysis of left bundle branch block. It was subsequently discovered and validated that in the LBBB subgroup, patients received substantial benefit from CRT-D. Non-LBBB patients did not show evidence of benefit. The LBBB subgroup made up approximately $70 \%$ of the total MADIT-CRT population (adapted from Boston Scientific CRT-D product labeling) 
ongoing genomic revolution and the trend toward personalized medicine - in the coming world, we may each comprise our own subgroup of one!

On the positive side, large-scale registries such as the ACC/NCDR ICD registry may provide long-term data for the analysis of outcomes [29]. These registries may provide a more cost-effective method for assessing the comparative effectiveness of therapies, particularly when coupled to remote patient monitoring. Unfortunately, this method does not provide an approach to assessing outcomes in an alternative treatment group.

Finally, an important question, again related to time, will be when to stop. When do policy makers and physicians determine that we have "enough" information? Without "stopping rules" for CER similar to those found in clinical studies, we run the risk of placing societal resources into efforts with ever diminishing marginal benefits. For example, is there a prospective plan of when to end the ACC/NCDR ICD registry? Unfortunately, stopping times will vary with each device type and will be particularly difficult to determine for innovative therapy, and there is a significant risk that pharmacologic principles may be inappropriately applied to devices. Particularly concerning is a recent publication by Chen et al. [34] which argues in favor of the "inclusion of comparative effectiveness data in high-risk cardiovascular device studies at the time of premarket approval." The implantable defibrillator, which represented innovative therapy in 1985, did not have a randomized comparator, and it required thoracotomy for lead and patch placement, and it is unclear whether it would have met this criteria for approval. However, clearly subsequent iterations of the ICD have demonstrated CER benefit, but without original approval, this benefit may never have been achieved. Similarly, the subcutaneous ICD did not have a randomized comparator group, but it is our opinion that this approach to pre-market CER would have been entirely inappropriate and significantly raised the regulatory burden to the point of potentially excluding beneficial therapy for high-risk cardiovascular patients for many of the previously cited reasons. The S-ICD which was approved by the FDA is a firstgeneration device which will rapidly be redesigned; physicians are currently unfamiliar with implantation techniques, and there will inevitably be a learning process for appropriate use. CER has a role later when there is stability of the device design, physician knowledge, and patient selection.

\subsection{Summary and a path forward}

The last half century has seen unprecedented improvements in health care with declines in cardiovascular deaths, due in part to the introduction of a series of revolutionary new medical technologies. Nevertheless, current circumstances pose significant hurdles to continuing medical device innovation: the regulatory environment is globally fragmented and currently particularly burdensome in the USA. We have a clear need for good comparative effectiveness data for new technologies, but there are particular issues with designing comparative clinical trials and interpreting CER assessments in the context of new medical devices. There clearly are very divergent views on this topic, and undoubtedly, there are even divergent views within industry. However, we believe that there are potential paths to maintain and improve innovation, as seen from our industry viewpoint:

Industry:

1. Greater care is needed in planning innovative products that meet today's health care needs. Where there is change, there is also new opportunity (e.g., reducing 30-day re-hospitalization for heart failure).

2. Product cadence should be coupled to available outcomes data (e.g., a new feature in a medical device should be planned to be submitted for approval shortly after there is beneficial clinical data to support that feature).

3. Developing and expanding methods such as remote patient monitoring to reduce the cost for CER and potentially to improve analysis of patient outcomes and device performance

4. Making more cost-effective devices. For example, a longer lasting device results in a lower cost over the long term. Market drivers in low- and middle-income nations bring this to a higher priority for industry.

5. Less invasive alternative innovative therapies not only may reduce costs by avoiding surgical procedures but may also reduce the costs related to surgical complications.

6. Researching the currently unmet needs in health care today (e.g., disease processes with disproportionate expenditure or poor outcomes currently such as heart failure with preserved ejection fraction)

7. Improved device reliability to reduce costs related to device malfunctions (e.g., current focus in CRM is on lead reliability)

8. Co-sponsored CER with government, other manufacturers, and include active participation from physician bodies

9. Develop genotypic or biomarker-based device indications with improved cost/benefit ratio

\section{Physicians:}

1. Set appropriate expectations amongst physicians, regulators, and patients about what realistic device performance should be and set up forums to publicly share this with industry. Physicians are encouraged to volunteer for advisory roles with FDA, CMS, and other payers and to express concern when they believe that regulatory barriers are excessive.

2. Physicians and industry need to disseminate understanding of the tensions between novel designs and 
reliability. Thus, when a physician requests a smaller $\mathrm{F}$ size lead, which may be easier to place in the patient, the industry design compromises may lead to potentially greater malfunction rates. In the case of an ICD lead, the net clinical effect has sometimes been suboptimal. In contrast, reducing the size of left ventricular coronary venous leads to $4 \mathrm{~F}$ has enhanced the usability significantly, but it is as yet unclear if there is a reliability trade-off in lead life. Similar compromises between functionality and reliability often apply to more complex device feature sets. Programming higher tachycardia detection rates may reduce inappropriate shocks from an ICD and may be beneficial, but in extremes may result in failure to detect ventricular fibrillation with low rates at the RV electrode.

3. There is a tendency by multiple parties to lay blame when device performance is not as expected in the long term. That is, when the manufacturer has performed the appropriate testing, consistent with engineering knowledge in place at the time of development, and that testing satisfies regulatory requirements, it is hard to know whether there is fault if the performance is suboptimal in the long term. Stated in other words, it is difficult to test for unknown failure mechanisms and mitigate unknowns, particularly in novel products and design. All parties need to consider how to address and educate on this, and physician bodies may choose to consider the malfunction rates found in post-approval studies when updating therapy guidelines.

4. Understanding the potential trade-off in design versus new features such as magnetic resonance conditional safety is important for physicians (e.g., devices do not sense during the scan, long-term confusion among nondevice following physicians about which patients are conditionally safe).

5. In dealing with devices with less than optimal performance, physician bodies need to carefully consider the risks of intervention and compare that with the risks of non-intervention in developing recommendations.

6. Physicians need to be vigilant for device malfunctions and report to both the FDA and the manufacturer. Returning products for manufacturer analysis where there is concern is important.

Government (legislative and regulatory bodies):

1. Efforts to produce regulatory convergence across borders

2. Consider reviewing the regulatory burden for device approvals. The FDA does have a fast pathway for innovative therapy with unmet health care need. However, even this pathway has a significant cost, as seen with the recent S-ICD development which took over a decade and cost in excess of 400 million dollars.
3. Maintain consistent expectations for product performance consistent with device design reality

4. Educate the public on the expected performance that is considered acceptable

5. Consider the use of less burdensome remote follow-up device data coupled to CMS or NCDR data to satisfy the post-market surveillance needs

6. Develop innovative payment policies to enable savings gained by one stakeholder using innovative technologies to be shared with other stakeholders

7. Develop an understanding that processes used in monitoring pharmaceuticals sold to millions of patients over a decade are difficult to apply to devices that are often sold in numbers $<100,000$ for 2 3 years for individual models

8. Provide incentives for greater cost effectiveness. For example, greater battery longevity is usually associated with larger device size, which is not popular with physicians or patients. The manufacturer potentially may lose money because of fewer generator replacements. The major beneficiary of this technology is the health care payer, but currently, they do not provide incentives for longevity.

9. Provide for reimbursement during an interim period after the development of a novel medical technology, such that the technology might be optimally explored and experience gained, before definitive CER studies are done. While this may exist in CPT coding, CMS may not always rule in concert with FDA approval.

10. A practical delineation of appropriate physician-industry collaboration to produce optimal future devices

Open Access This article is distributed under the terms of the Creative Commons Attribution License which permits any use, distribution, and reproduction in any medium, provided the original author(s) and the source are credited.

\section{References}

1. Moore, R. (1966). Niels Bohr: the man, his science, \& the world they changed, p. 196. Retrieved 13 April 2012 from http://en.wikiquote.org/ wiki/Niels_Bohr.

2. Greg, E. W., Cheng, Y. J., Saydah, S., Cowie, C., Garfield, S., \& Geiss, L. (2012). Trends in death rates among U.S. adults with and without diabetes between 1997 and 2006. Diabetes Care, 35, 1252-1257.

3. Roger, V. L., Go, A. S., Lloyd-Jones, D. M., Adams, R. J., Berry, J. D., Brown, T. M., et al. (2011). Heart disease and stroke statistics - 2011 update: a report from the American Heart Association. Circulation, 123 , e18-e209.

4. U.S Senate, Special Committee on Ageing (2011) Hearing examining the Food and Drug Administration's (FDA) role in protecting patient safety as part of the medical device approval process, 13 April 2011. Retrieved 29 May 2012 from aging.senate.gov/hearing detail.cfm?id=332494\&. 
5. Illinois Plaintiff's Lawyer. Defective products, especially prostheses are not always recalled. Retrieved 29 May 2012 from illinoisplaintiffslawyer.com/?p=64.

6. Holmes, D. R., \& Wood, D. L. (2006). Medical devices and pharmaceuticals: differences in public acceptance. The American Heart Hospital Journal, 4(4), 269-272.

7. Overview of Device Regulation. Retrieved 16 October 2012 from http://www.fda.gov/Medical Devices/Device Regulation and Guidance/Overview/default.htm.

8. European Standards. Retrieved 17 October 2012 from http:// www.ec.europa.eu/enterprise/policies/european-standards/ harmonized-standards/implantable-medical-devices/index_en.htm.

9. DeMaria, A.N. (2009). The exportation of clinical research. Retrieved 29 May 2012 from theheart.org/documents/ sitestructure/en/content/1061541/demaria.html.

10. Global Harmonization Task Force Summary technical documentation for demonstrating conformity to the essential principles of safety and performance of medical devices (STED). Retrieved 29 May 2012 from ghtf.org/documents/sg1/sg1 final-n11.pdf.

11. FDA. Retrieved 16 October 2012 from http://www.fda.gov/ RegulatoryInformation/RulesRegulations/default.htm.

12. EPA. Retrieved 16 October 2012 from http://www.epa.gov/ lawsregs/basics.html/\#regulation.

13. FDA. Retrieved 16 October 2012 from http://www.fda.gov/ RegulatoryInformation/Legislation/FederalFoodDrugandCosmetic ActFDCAct/SignificantAmendmentstotheFDCAct/FDASIA/ ucm20027187.htm.

14. Maisel, W. H., Moynahan, M., Zuckerman, B., Gross, T. P., Tovar, O. H., Tillman, D.-B., et al. (2006). Pacemaker and ICD generator malfunctions: analysis of food and drug administration reports. JAMA: The Journal of the American Medical Association, 295, 1901-1906.

15. Laskey, W., Awad, K., Lum, J., Skodacek, K., Zimmerman, B., Selzman, K., et al. (2012). An analysis of implantable cardiac device reliability. The case for improving postmarketing risk assessment and surveillance. American Journal of Therapeutics, 19, $248-254$.

16. Draft Guidance for Industry and Food and drug Administration Staff-Investigational device exemptions (IDE) for early feasibility medical device studies, including certain first in human (FIH) studies. Retrieved 29 May 2012 from fda.gov/MedicalDevices/ DeviceRegulationandGuidance/GuidanceDocuments/ucm 277670.htm.

17. Segupta, J., Kendig, A. C., Goormastic, M., Hwang, E.-S., Ching, E. A., Chung, R., et al. (2012). Implantable cardioverterdefibrillator FDA safety advisories: impact on patient mortality and morbidity. Heart Rhythm, 9, 1619-1626.

18. Carlson, M. D., Wilkoff, B. L., Maisel, W. M., Ellenbogen, K. A., Saxon, L. A., Prystowsky, E. N., et al. (2006). Recommendations from the Heart Rhythm Society Task Force on Device Performance Policies and Guidelines. Heart Rhythm, 3, 1250-1273.

19. Maisel, W. M. (2004). Physician management of pacemaker and implantable cardioverter defibrillator advisories. Pacing and Clinical Electrophysiology, 27, 437-442.

20. Gould, P. A., \& Krahn, A. D. (2006). Canadian Heart Rhythm Working Group on Device Advisories. Complications associated with implantable cardioverter-defibrillator generator replacement in response to device advisories. JAMA: The Journal of the American Medical Association, 295, 1907-1911.

21. Medtronic. Retrieved 16 October 2012 from http://wwwp. medtronic.com/productperformance/.

22. Makower, J. (2010). FDA impact on U.S. medical technology innovation. Retrieved 29 May 2012 from advamed.org/NR/ rdonlyres/040E6C33-380B-4F6B-AB58-9AB1C0A7A3CF/0/ makowerreportfinal.pdf.
23. Maisel, W.H. Premarket notification analysis of FDA recall data. Retrieved 29 May 2012 from iom.edu/ /media/Files/Activityfiles/ PublicHealth/510kProcess/2010-Jul-28/05/Maisel.pdf.

24. For a variety of reasons, Congress chose not to fund the OTA starting after September 30, 1995. See http://www.princeton.edu/ ota/ for more information about the OTA.

25. PCORI funding. Retrieved 16 October from http://www.pcori.org/ how-were-funded/.

26. NIH funding. Retrieved 16 October 2012 from http://nih.gov/ about/budget.htm.

27. Salukhe, T. V., Dimopolous, K., Sutton, R., Coats, A. J., Piepoli, M., \& Francis, D. P. (2004). Life-years gained from defibrillator implantation: markedly nonlinear increase during 3 year follow-up and its implications. Circulation, 109, 1848-1853.

28. Goldenberg, I., Gillespie, J., Moss, A. J., Hall, W. J., Klein, H., McNitt, S., et al. (2010). Long-term benefit of primary prevention with an implantable cardioverter-defibrillator: an extended 8-year follow-up study of the Multicenter Automatic Defibrillator Implantation Trial II. Circulation, 122, 1265-1271.

29. Bilchick, K. C., Stuckenborg, G. J., Kamath, S., \& Cheng, A. (2012). Prediction of mortality in clinical practice for Medicare patients undergoing defibrillator implantation for primary prevention of sudden cardiac death. Journal of the American College of Cardiology, 60, 1647-1655.

30. Reddy, V. Y., Holmes, D., Doshi, S. K., Neuzil, P., \& Kar, S. (2011). Safety of percutaneous left atrial appendage closure: results from the Watchman Left Atrial Appendage System for Embolic Protection in Patients with AF (PROTECT AF) Clinical Trial and the Continued Access Registry. Circulation, $123,417-424$.

31. Pacemakers and implantable defibrillators: UK National Survey. Retrieved 16 October 2012 from http://www.devicesurvey.com.

32. Scott, P. A., Turnes, N. G., Chugh, A., Morgan, J. M., \& Roberts, P. P. (2009). Varying implantable cardioverter defibrillator referral patterns from implanting and non-implanting hospitals. Europace, $11,1048-1051$.

33. Mack, M. J., \& Holmes, D. R. (2011). Rational dispersion for the introduction of transcatheter valve therapy. JAMA: The Journal of the American Medical Association, 306, 2149-2150.

34. Chen, C. E., Dhruva, S. S., \& Redberg, R. F. (2012). Inclusion of comparative effectiveness data in high-risk cardiovascular device studies at the time of premarket approval. JAMA: The Journal of the American Medical Association, 308, 1740-1742.

35. Wever, E. F. D., Hauer, R. N. W., van Capelle, F. J. L., et al. (1995). Randomized study of implantable defibrillator as firstchoice therapy versus conventional strategy in post-infarct sudden death survivors. Circulation, 91, 2195-2203.

36. Moss, A. J., Hall, W. J., Cannom, D. S., et al. (1996). Improved survival with an implantable defibrillator in patients with coronary disease at high risk for ventricular arrhythmia: the Multicenter Automated Defibrillator Implantation Trial (MADIT) Investigators. The New England Journal of Medicine, 335, 1933-1940.

37. The Antiarrhythmics Versus Implantable Defibrillators (AVID) Investigators. (1997). A comparison of antiarrhythmic drug therapy with implantable defibrillators in patients resuscitated from near fatal ventricular arrhythmias. The New England Journal of Medicine, 337, 1576-1583.

38. Connolly, S. J., Gent, M., Roberts, R. S., et al. (2000). Canadian Implantable Defibrillator Study (CIDS): a randomized trial of the implantable cardioverter defibrillator against amiodarone. Circulation, 101, 1297-1302.

39. Moss, A. J., Zareba, W., Hall, W. J., et al. (2002). Prophylactic implantation of a defibrillator in patients with myocardial infarction and reduced ejection fraction: the Multicenter Automated 
Defibrillator Implantation Trial II (MADIT II) Investigators. The New England Journal of Medicine, 346, 877-883.

40. Kuck, K.-H., Cappato, R., Siebels, J., et al. (2000). Randomized comparison of antiarrhythmic drug therapy with implantable defibrillators in patients resuscitated from cardiac arrest: the Cardiac Arrest Study Hamburg (CASH). Circulation, 102, 748-754.

41. Schläpfer, J., Rapp, F., Kappenberger, L., et al. (2002). Electrophysiological guided amiodarone therapy versus the implantable cardioverter-defibrillator for sustained ventricular tachyarrhythmias after myocardial infarction. Journal of the American College of Cardiology, 39, 1813-1819.

42. Buxton, A. E., Lee, K. L., Fisher, J. D., et al. (1999). A randomized study of the prevention of sudden death in patients with coronary artery disease: the Multicenter Unsustained Tachycardia Trial (MUSTT) Investigators. The New England Journal of Medicine, $341,1882-1890$. 